\title{
Comment éduquer à la fraternité par la pratique de la discussion à visée philosophique dans l'école de la République?
}

How to educate to fraternity thanks to philosophical discussion in French republican schools?

\section{Christian Budex}

\section{(Q) OpenEdition \\ Journals}

\section{Electronic version}

URL: http://journals.openedition.org/trema/5776

DOI: 10.4000/trema.5776

ISSN: 2107-0997

\section{Publisher}

Faculté d'Éducation de l'université de Montpellier

\section{Electronic reference}

Christian Budex, «Comment éduquer à la fraternité par la pratique de la discussion à visée

philosophique dans l'école de la République ? », Tréma [Online], 53 | 2020, Online since 01 March 2020, connection on 03 March 2020. URL : http://journals.openedition.org/trema/5776 ; DOI : 10.4000/

trema.5776

This text was automatically generated on 3 March 2020.

Trema 


\title{
Comment éduquer à la fraternité par la pratique de la discussion à visée philosophique dans l'école de la République?
}

\author{
How to educate to fraternity thanks to philosophical discussion in French \\ republican schools?
}

Christian Budex

\section{Introduction : la fraternité, une notion à géométrie variable}

Dernière marche du «divin perron » républicain selon Hugo, la fraternité reste la parente pauvre de la triade « liberté-égalité-fraternité » et n'a pas eu l'honneur d'être l'objet d'analyses aussi nombreuses et approfondies que ses deux célèbres consœurs (Budex, 1999). Souvent considérée comme une notion vague, un signifiant flottant, une intuition sans concept, elle n'a pas été épargnée par les critiques qui lui reprochent à la fois son sentimentalisme, son manque de maniabilité politique, et de façon générale d'être plus affective qu'effective ${ }^{3}$. Elle peut à l'inverse être comprise comme un concept performatif, une force "idéomotrice " (Bergson, 2012, p. 283), un sentiment d'action subversif indispensable à l'équilibre du triptyque républicain et par là-même à toute société démocratique ${ }^{4}$. Au-delà de son inscription politique dans l'histoire française depuis la Révolution, elle est un nœud épistémologique situé au carrefour de la plupart des sciences humaines et dont le mérite est d'interroger la place décisive des affects en politique à partir de la question du sentiment d'appartenance. Elle est aussi une notion ambivalente, un pharmakon ${ }^{5}$ à géométrie variable dont on pourrait dire, pour parodier Claude Lévi-Strauss, qu'elle "pose immédiatement deux problèmes qui sont, si j'ose dire, ceux de son emploi au singulier et au pluriel » (Lévi-Strauss, 1983, p. 55). 
2 On peut repérer d'une part, la "grande» fraternité du genre humain. J'appelle fraternité humaniste le sentiment d'appartenance à une famille d'êtres vivants dont les frontières - poreuses, variables selon les représentations, et même contestables en droit - sont de fait suffisamment bien établies pour constituer une identité qui conduit les humains à reconnaître la dignité de tout humain, quelques soient ces particularismes. La fraternité interroge alors la dimension affective de ce lien qui unit les humains au travers des expériences de l'altérité qui permettent de l'éprouver et dont les occurrences philosophiques modernes sont bien connues : la compassion ou la sympathie chez Adam Smith, la pitié chez Rousseau, la dignité chez Kant, l'hospitalité (Derrida, 1997), le tact (Prairat, 2017) ou l'empathie 6 pour les recherches les plus récentes. Dans cette première dimension interindividuelle, disons éthique et/ou morale ${ }^{7}$, elle est à la fois un principe et une valeur ${ }^{8}$ dont nos sociétés ont besoin pour faire face aux replis identitaires et aux discriminations de tous ordres.

3 Il existe, d'autre part, des fraternités plus petites, plus ou moins ouvertes et plus ou moins fraternelles : celles des sociétés, des groupes, des confréries formés par ceux qui se reconnaissent comme frères, comme membres de la même famille, parce qu'ils partagent les mêmes principes, les mêmes idéaux, voire les mêmes combats, à partir de critères autonomes ou hétéronomes, qu'ils soient ethniques (les kabyles ou les bretons), religieux (les chrétiens ou les musulmans), idéologiques (les communistes), sociaux (les classes ou les ordres), voire professionnels (les confréries de métier depuis le Moyen Âge). Dans cette dimension plus identitaire, disons politique ou sociale, la fraternité se conjugue alors au pluriel. Le champ d'étude de ces fraternités communautaires devient celui des logiques d'appartenance et de reconnaissance à l'intérieur d'un groupe ${ }^{9}$.

Avant d'être importée dans le champ sociologique, philosophique, juridique, la notion de fraternité renvoie d'abord à un lien biologique, celui qui désigne la relation familiale qui unit les frères ou les sœurs de sang. Ce n'est que par une extension métaphorique, et en un sens plus large, qu'elle en est venue à désigner l'affection qui unit des hommes qui se reconnaissent comme membres d'une même famille identitaire. Ainsi la notion de fraternité fonctionne comme une « métaphore vive » (Ricœur, 1983) et reste habitée par sa structure logique d'origine: celle d'une relation entre des individus qui partagent la même filiation. Elle nous conduit donc à interroger le sentiment d'appartenance dans son double lien d'horizontalité et de verticalité.

\section{Logique d'appartenance d'une fraternité sans dieux}

\section{Verticalité, sacralité et transcendance}

$5 \quad$ Il n'y a pas de fraternité sans une parenté commune et pas de lien horizontal sans une attache verticale qui a pour fonction de maintenir ce lien. Traduit dans le champ social et hors du domaine de la famille, cela signifie que ce qui fait du lien entre les hommes est à chercher dans du supérieur sacré commun qui les dépasse et les attache. Première leçon de Rousseau, Comte et Durkheim ${ }^{10}$ : c'est la sacralité qui crée du lien, le respect commun de quelque chose - dieu, entité surnaturelle, autorité - qui est "mis à part ", " séparé » (secernere en latin) et auquel on reconnaît une valeur supérieure, intangible. S'il y a bien une dimension sociale de la religion parce qu'elle relie (religare en latin) les hommes qui partagent les mêmes croyances, il y a également une dimension religieuse 
du lien social puisque toute société qui aspire à faire corps a besoin de se référer à une transcendance symbolique.

«Pour produire de l'inter, donnez-nous du meta (Debray, 2005, p. 86). Tout se passe comme s'il existait un invariant anthropologique et sociologique qui attache et relie plus ou moins fortement les individus par des liens horizontaux à partir d'une sacralité verticale. On peut ainsi repérer les principaux archétypes qui servent de sacralité d'attachement, de meta, et qui soudent le multiple à l'Un: Dieu et la religion, le mythe, l'idéal, l'idéologie, l'Histoire (ou plutôt la mémoire) et l'un des plus redoutables, dont la transcendance est très faible mais qui nourrit un imaginaire puissant et efficace: l'ennemi commun. On peut même dégager une typologie, voire une axiologie des transcendances à partir de laquelle on peut évaluer les bénéfices sociaux, moraux, politiques de tel ou tel meta, tout comme aussi leur puissance d'adhésion.

Bien sûr, cette logique d'appartenance est complexe et on observe des groupes dont le meta d'ancrage est un mixte de plusieurs archétypes. Ainsi le « patriotisme » américain repose sur un mixte relativement bien équilibré de meta religieux (essentiellement chrétien, à dominante protestante), idéal (le « rêve américain »), historique (culte des anciens et du drapeau). Le sentiment d'appartenance en Israël, quant à lui, repose sur un autre mixte de meta religieux (version ancien Testament) et mythique (la terre promise, le peuple élu).

8 Cette psychosociologie de l'appartenance peut alors nous reconduire à l'Histoire, en l'occurrence celle de la République en France. En observant l'acte de naissance de Marianne, on constate en effet que le moment révolutionnaire ne se caractérise pas, malgré sa libération à l'égard du joug paternel de la royauté, par l'abandon de toute filiation, mais par un changement de filiation et d'autorité : on passe d'une logique de la soumission au père à une structure d'obéissance à la mère, du Roi à la République, du droit divin à la loi citoyenne. Pour sacraliser sa nouvelle structure politique, la Révolution, plus ou moins consciemment, s'est donc tournée vers des figures maternelles : la République, la Loi, la Patrie, la Nation. Ces nouvelles sacralités drainent avec elles leurs sentiments corollaires, pour le meilleur et pour le pire et du plus froid au plus chaud : civisme, patriotisme, nationalisme.

9 Le problème est qu'un ordre politique qui lie les hommes par l'autorité de la loi fait peut-être des frères de droit, mais pas nécessairement des frères de cœur. La loi est une figure abstraite, peu sensible, et la citoyenneté est une appartenance juridique qui peine, à elle seule, à créer une affection commune et un véritable sentiment d'appartenance.

\section{Une nouvelle filiation problématique}

10 L'entreprise est d'autant plus délicate que, depuis la Révolution, le processus de sécularisation, puis de laïcisation, a peu à peu conduit la France à rompre avec l'une des sacralités les plus liantes - Dieu et la Religion - au profit d'un mixte de trois meta : l' Histoire et/ou la Mémoire de la Révolution, l'idéal de son texte fondateur (la Déclaration universelle des droits de l'homme et du citoyen de 1789), voire le mythe de la République. Telle est la nouvelle donne d'une logique d'appartenance pour une fraternité sans dieux. La difficulté est même redoublée du fait qu'en vertu de son idéal, la fraternité révolutionnaire, dans sa version la plus ouverte, ouvre les bras vers l'universel et l'humanisme. Reste le problème de l'amplitude ${ }^{11}$ : lorsqu'elle embrasse trop large, la fraternité perd en intensité de lien, comme si son sentiment faiblissait par 
manque de reconnaissance identitaire; on perd alors en compréhension ce que l'on gagne en extension. Rousseau opposait ainsi le problème de l'efficience politique aux " saines idées du droit naturel et de la fraternité commune à tous les hommes ${ }^{12}$ » :

Il semble que le sentiment de l'humanité s'évapore et s'affaiblisse en s'étendant sur toute la terre, et que nous ne saurions être touchés des calamités de la Tartarie ou $\mathrm{du}$ Japon, comme de celles d'un peuple européen. Il faut en quelque manière borner et comprimer l'intérêt et la commisération pour lui donner de l'activité ${ }^{13}$.

11 Une société pourrait donc souffrir d'un déficit d'appartenance et de fraternité parce qu'elle est arrimée à un meta dont l'horizon universaliste détermine très, voire trop largement son spectre identitaire. Tel est le problème franco-français d'une fraternité qui est à la fois, dans sa devise et son histoire, nationale et universelle - du moins dans son idéal -, et qui se présente de manière paradoxale comme un appel citoyen à l'humanisme. Il y a des sacralités d'attachement plus ou moins efficaces pour souder un peuple et le choix historique de la France pour un meta ouvert, juridico-philosophique et laïque ne facilite pas la tâche.

On comprend mieux pourquoi la fraternité, comme une cellule fragile, tend trop souvent à se replier en communauté pour s'éprouver véritablement, comme si la différence et l'adversité soudaient davantage que l'identité et la concorde. Telle est la part d'ombre de la fraternité : elle porte en elle une logique d'inclusion / exclusion " mortifrère » qui fabrique du «faux-frère » et de la "frérocité » en un rien de temps. Au mieux, les individus se recroquevillent sur la fraternité plus chaude des agrégations communautaires, qu'elles soient ethniques ou religieuses, tandis que la fraternité citoyenne, version drapeau national, est en berne; au pire, nous assistons à l'atomisation sociale des individualismes juxtaposés. On constate alors dans la société un effet centrifuge là où l'on espérait un effet centripète. Il y aurait un différentiel entre citoyenneté et fraternité qui fait défaut à la République dans la mesure où la première fait communauté sans faire communion. Et si la solidarité peut bien avoir son utilité socioéconomique, elle ne génère pas de sentiment d'appartenance.

13 Toutefois, si l'ancrage de tout groupe à une sacralité verticale est une condition nécessaire pour comprendre la nature et l'intensité du lien qui en unit les membres, elle n'est pas suffisante pour autant.

\section{Horizontalité, culte et culture}

14 Après avoir interrogé l'élaboration d'une fraternité à partir de son ancrage vertical, il convient également d'interroger sa dimension horizontale. Pour comprendre comment «transmuer un tas en tout " (Debray, 2009, p. 278), il nous faut donc aller voir du côté des champions de la fraternité, à commencer par les confréries religieuses. Si toute religion peut être considérée comme une secte qui a réussi, est-il possible de demander par quels moyens? Quelles sont les recettes de l'adhésion fraternelle ? Jusqu'où peutelle s'étendre et à quelle logique obéit-elle?

Seconde leçon de Rousseau, Comte et Durkheim: le lien de sacralité qui relie tout groupe à une transcendance s'entretient et se cultive.

Il ne peut pas y avoir de société qui ne sente le besoin d'entretenir et de raffermir, à intervalles réguliers, les sentiments collectifs et les idées collectives qui font son unité et sa personnalité. Or, cette réfection morale ne peut être obtenue qu'au moyen de réunions, d'assemblées, de congrégations où les individus, étroitement rapprochés les uns des autres, réaffirment en commun leurs communs sentiments ; 
de là, des cérémonies qui, par leur objet, par les résultats qu'elles produisent, par les procédés qui y sont employés, ne diffèrent pas en nature des cérémonies proprement religieuses. (Durkheim, 1912, p. 610) fraternité, defficacité lidéal des droits de l'homme et du triptyque liberté, égalité, fraternité, l'histoire ou la mémoire de ses grands personnages et de ses grands événements. Empruntant leur déroulement aux logiques des pratiques religieuses, les commémorations et les cérémonies collectives ont bien pour fonction de lier les individus par le respect commun d'un domaine sacré composé par les héros, les dates, les lieux, les événements ou les textes importants qui constituent à la fois la mémoire collective et les symboles d'une communauté. «La France est une religion» (Michelet, 1877, p. 272). Comprenons bien : il y a une dimension religieuse du lien social dans la mesure où toute société a besoin d'arrimer son collectif à une sacralité d'ancrage pour rester soudée. Et qui dit sacralité dit culte, c'est-à-dire un ensemble de pratiques qui permettent, comme le rappelle Durkheim, «d'entretenir et de raffermir, à intervalles réguliers, les sentiments collectifs et les idées collectives ", (Durkheim, 1912, p. 60). Rousseau avait bien perçu cette nécessité de donner un souffle de vie au corps politique. Pour cela, il a conçu l'étrange idée d'une «religion civile» dont la fonction est de faire apparaître des «sentiments de sociabilité » (Rousseau, 1762, p. 179), de susciter une implication affective, un attachement sensible au bien commun ou à la chose publique.

\section{Veiller à la température de la fièvre groupale}

Les principes sont posés et la recette connue : un peuple qui fait corps suppose des sacralités et des cultes qui permettent d'entretenir le lien vertical d'autorité et le lien horizontal d'appartenance. Toutefois, comme dans toute recette, tout est affaire de dosage; il faut trouver la bonne température de la «fièvre groupale " pour parvenir à un juste milieu social entre le point de fusion intégriste et le zéro absolu de l' individualisme atomisé. Que la température de la communion soit trop faible, et voilà le sentiment d'appartenance qui se dissout dans l'isolement. Mais si le culte de l'idéal vire à l'idéologie parce que les politiques jouent sur le vibrato sensible de la Nation, alors c'est le retour de "la religion drapeautique ", comme l'appelle Bardamu, le héros célinien du Voyage au bout de la nuit. L'idéologie, source de toutes les pathologies fraternitaires en -isme (nationalisme, fascisme, stalinisme, maoïsme, etc.) est un adhésif efficace, mais c'est un précipité instable qui sent la poudre et l'exclusion.

Anticipant les dérives d'un culte rendu à la Nation, Rousseau avait d'ailleurs prudemment pris le soin de bien distinguer « la religion civile » de ce qu'il nomme «la religion du citoyen "; l'utilité de cette dernière est bien d'attacher les citoyens aux lois, mais elle a aussi pour inconvénients de considérer tout ce qui est étranger à la nation comme barbare et de ne définir des devoirs qu'à l'égard des concitoyens, non à l'égard 
de l'ensemble de l'humanité. Elle est en quelque sorte l'inverse du Christianisme, qui étend ses devoirs à l'humanité entière mais qui, selon Rousseau, se rend incapable de défendre sa patrie. On retrouve le problème de l'amplitude et les limites de toute fraternité : si elle est trop ouverte, elle embrasse sans étreindre, mais si l'on veut un lien plus fort, on court le risque de fabriquer de l'exclusion. De l'universalisme au communautarisme, la fraternité s'étiole ou isole.

Malgré ses défauts, l'idée de religion civile constitue une ébauche d'incarnation institutionnelle du principe de fraternité. Peut-on sérieusement penser l'avenir d'une religion civile en France? La proposition paraît indécente, tant l'association entre religion et politique semble sulfureuse depuis la séparation de l'Église et de l'État en 1905.

\section{La fraternité ou l'affect du triptyque : comment l'incarner?}

21 Tardivement reconnue comme indispensable à la solidité de l'édifice, la fraternité semble, contrairement à ses deux consœurs, difficile à incarner concrètement dans la réalité sociale, politique et surtout juridique (Borgetto, 1993). Elle occupe donc une place à part dans la famille. Parce qu'elle se situe plutôt du côté de la morale et des sentiments, elle échappe par là même au domaine de l'action politique alors qu'elle en constitue pourtant une condition : pour qu'un collectif puisse perdurer, il doit pouvoir compter sur une certaine dose d'adhésion affective. S'il ne peut y avoir de fraternité sans garantie juridique de la liberté et de l'égalité, il n'est pas vrai pour autant que l'assise juridique de ces deux valeurs suffise à maintenir la cohésion du tout. Il ne pourrait y avoir de chose publique sans un cœur commun. La fraternité, c'est l'indispensable pathos qui donne sa vie au Tout et nous rappelle qu'une société est un organisme vivant doué d'affects.

Toutefois, les difficultés commencent lorsqu'on tente de donner vie à ce sentiment dans des pratiques. Comment pourrait-on décréter un sentiment ou légiférer sur l'affectif? Michelet a bien résumé cette quadrature du cercle :

a/ si la fraternité est laissée au sentiment, elle n'est pas efficace, ou elle l'est pour une heure d'élan ;

b/ si elle est écrite en loi et impérative, elle n'est plus fraternelle ;

c/ si vous voulez qu'elle s'étende il faut qu'elle soit volontaire, et alors nous retournons au sentiment que nous avons quitté, (Journal du 11 février 1847).

Si elle ne peut se décréter, n'est-il pas possible de la sécréter, c'est-à-dire d'en permettre l'émergence en favorisant ses conditions de possibilité ? Si la fraternité ne peut advenir par le fait d'une loi impérative, ne faut-il pas plutôt la confier à l'éducation?

\section{L'éducation à la fraternité dans l'école de la République}

\section{Comment éduquer à la fraternité ?}

L'École de la République a, dès son origine, pensé la nécessité d'une instruction ou d'une éducation pour tenter de développer des dispositions à agir qui produisent des comportements "civiques" et favorisent ce qui s'appelle aujourd'hui, dans une formule éprouvée, le "vivre-ensemble ». Une telle entreprise s'est toutefois heurtée à 
la difficulté de passer d'un simple enseignement théorique à une véritable éducation, c'est-à-dire au développement progressif, mais effectif, de dispositions pratiques. Si elle a permis de faire lever les yeux en direction des frontispices, l'éducation à la citoyenneté n'a toutefois pas jusqu'ici véritablement réussi à faire naître une fraternité, à peine un civisme.

Nos temps troublés et menacés par la désunion réclament aujourd'hui davantage. L'actualité tragique des attentats de l'année 2015 semble avoir fait redécouvrir à la France la nécessité d'une fraternité républicaine autour de ses valeurs. On s'est alors presque naturellement tourné vers l'École pour la prier d'éduquer, voire de rééduquer au plus vite des élèves en mal d'adhésion, les français découvrant au passage que nombre de leurs « enfants » peinaient à partager l'idéal républicain. Ces attentats ont été l'occasion, dans l'école, d'une prise de conscience d'un déficit du sentiment d'appartenance considérable, et même d'une rupture de confiance civique entre les élèves, entre les élèves et les adultes de la communauté éducative, voire entre les adultes eux-mêmes. Une " grande mobilisation pour les valeurs de la République » s'est déclinée dans l'école en onze mesures ${ }^{14}$. Des formations sont nées pour tenter de « faire vivre " les valeurs de la République. L'émotion est passée, tout comme le calendrier politique, mais pas la nécessité d'interroger ensemble la valeur des valeurs pour tenter de donner ou redonner du sens à celles-ci.

L'entreprise reste toutefois délicate : il s'agit de susciter une adhésion en évitant le conformisme de façade d'un "catéchisme républicain descendant» dont beaucoup d'enseignants et d'éducateurs comprennent aujourd'hui qu'il est inefficace, voire contre-productif. On peut imposer une minute de silence au lendemain d'un attentat, on peut même sanctionner ceux qui ne la respectent pas, mais on n'impose pas plus le respect du sacré que l'adhésion aux valeurs. Il faut donc penser, dans l'école, une éducation et une pédagogie de la fraternité pour opposer l'idéal républicain aux forces de dégradation du nihilisme contemporain.

En quoi peut bien consister une telle éducation? Si elle ne peut être seulement théorique, quelles sont les pratiques qui permettent de susciter puis de cultiver le sentiment d'appartenance aux valeurs communes de la démocratie et de la République dans le moment même où les futurs citoyens s'insèrent dans le processus de socialisation? Comment penser des pratiques éducatives d'adhésion qui soient au service d'une culture de la fraternité sans pour autant servir une idéologie ? Comment créer une fraternité dans une société arrimée à un meta universel et laïque dont la sacralité est bien réelle, mais qui exige un culte et une culture particulièrement exigeants? Comment rendre sensible et favoriser un lien qui n'est d'abord qu'une abstraction juridique pour susciter du sentiment là où il n'y a d'abord que du droit ? Tel est l'enjeu d'une éducation ou d'un enseignement moral et civique dont nous voudrions interroger les conditions de possibilité. Le programme d'EMC, pensé avant les attentats mais mis en œuvre à la rentrée $2015^{15}$, recèle des pistes d'actions pour un tel défi éducatif.

\section{L'éducation aux valeurs dans le cadre du programme d'Enseignement Moral et Civique (EMC)} de conduire nos élèves à questionner et éprouver la dimension collective de leur 
existence dans le but de "transmettre" et "faire partager les valeurs de la République ». Or nous avons dit qu'un sentiment d'appartenance ne se décrète pas ; si "faire partager" signifie susciter une adhésion, voire un sentiment d'appartenance, alors il apparait qu'une telle entreprise n'est possible que par une prise de risque, un pari sur la valeur des valeurs qui conduit à accepter d'en interroger régulièrement les fondements pour les réassurer et espérer les rendre désirables. Il faut favoriser un cheminement intérieur d'adhésion par l'expérience même de ces valeurs si l'on veut effectivement les « transmettre » et les « faire partager ».

Comme le rappelait le Rapport sur l'enseignement laïque de la morale ${ }^{16}$ de 2013 qui a précédé le programme de 2015 , le pari pédagogique d'une véritable éducation citoyenne ne pourra être gagné qu'à condition de prendre en charge la triple dimension des valeurs: intellectuelle, psycho-affective, conative ${ }^{17}$. Il faut donc développer des dispositifs qui permettent d'interroger, d'éprouver et de faire vivre en acte ces valeurs. Le récent programme semble en effet tirer les leçons du passé en rompant avec le modèle vertical, normatif et injonctif d'une morale du devoir qui n'est plus en adéquation avec des sociétés démocratiques caractérisées par le pluralisme des opinions et des croyances. S'il ne renonce pas à valoriser un certain modèle de vie et de conduites par le biais d'un " perfectionnisme faible " (Kahn, 2016, p. 96), il entend promouvoir « une morale qui se construit de façon dialogique, dont les valeurs et les normes sont impliquées, mises en jeu, dans les situations de confrontations des idées et l'échange » (Kahn, 2016, p. 96).

31 Ce programme inaugure par ailleurs un nouveau paradigme anthropologique et moral dans lequel une éducation à la fraternité prend tout son sens. Il rompt en effet avec une conception trop intellectualiste pour reconnaitre l'importance de la vie affective dans l'éducation morale et civique au travers de l'appel à une culture de la sensibilité ; il insiste également sur la nécessité de faire vivre les valeurs par l'appel à une culture de 1 'engagement ${ }^{18}$. Autant d'éléments qui signalent le retour des affects dans le champ social et politique et qui semblent sonner l'heure d'une culture de la fraternité en prenant à rebours un reproche qui a été très souvent fait à cette notion : celui d'être plus affective qu'effective.

\section{Le rôle des affects dans les champs politique et éducatif}

On assiste depuis quelques années à une entrée de l'affectivité dans le champ des sciences, aussi bien dans le domaine des neurosciences (Damasio, 2014) que dans celui des sciences humaines, notamment politiques (Revaut d'Allones, 2004; Nussbaum, 2011 ; Lordon, 2016). Cet intérêt pour le rôle des affects, notamment dans la compréhension du champ politique, peut changer le regard sur une notion - la fraternité - qui porte en elle « un important potentiel en termes de réémotionalisation de la relation politique sécularisée, rationalisée et désenchantée " (Ménissier, 2012, p. 42). Un certain nombre de travaux ont déjà commencé à tirer les conclusions, dans le domaine éducatif, de cette nouvelle approche anthropologique en s'intéressant au rôle des émotions et de l'empathie (Tisseron, 2010 ; Catheline, 2015 ; Zanna, 2015 ; Guegen, 2018), tant dans le champ cognitif de l'entrée dans les apprentissages, que dans celui, relationnel, de la prévention des violences et de l'amélioration du climat scolaire (Bidar, 2015 ; Marsollier, 2016). 

reconnaissance des émotions, des sentiments, il semble encore incertain et timide à propos de la fraternité. Dans l'une de ses trois finalités - « respecter autrui »- il invite certes à " développer avec lui des relations de fraternité ». Pour le cycle 3, la fraternité est bien reconnue comme une "valeur " qu'il faut " expliquer en mots simples » - en compagnie de l'inséparable solidarité ${ }^{19}$-. Le programme du cycle 4 propose quant à lui d'« exprimer des sentiments moraux", de réfléchir au "sentiment d'appartenance à l'échelle d'un État", de "comprendre la diversité des sentiments d'appartenances civiques, sociaux, culturels, religieux» ainsi que "le sentiment d'appartenance au destin commun de l'humanité ", mais sans nommer la fraternité. Le programme du lycée ${ }^{20}$, dans son préambule, considère l'EMC « comme un temps d'apprentissage et de réflexion sur ce qui fonde la relation à l'autre dans une société démocratique ", (BO spécial $n^{\circ} 8$ du 25 juillet 2019 , p. 2) ; il doit permettre, au travers des trois thématiques retenues, - la liberté, la société, la démocratie - «d'aborder le sens et la portée des valeurs de la devise républicaine ». On n'y trouve pourtant aucune occurrence de la notion de "fraternité ». Seule la notion de «solidarité » est considérée comme l'une des notions à acquérir dans le programme de Première. Déjà le rapport de 2013 sur l'enseignement laïque de la morale ignorait la fraternité. Tout se passe comme si on ne savait quoi faire avec cette valeur si souvent négligée ${ }^{21}$ alors que de nombreux indicateurs ${ }^{22}$ nous montrent qu'il est urgent de développer une culture de la fraternité.

\section{L'éducation à la fraternité par la pratique de la philosophie à l'école}

Si un travail sur les compétences sociales et émotionnelles, en particulier l'empathie, constitue un élément déterminant d'une prévention des violences et d'une éducation heureuse (Gueguen, 2018), il faut y ajouter une éducation plus approfondie de l'altérité pour conduire les élèves à un apprentissage de la vie sociale qui puisse appréhender, en théorie comme en pratique, la dimension collective de leurs différentes appartenances : la classe, l'établissement, la famille, les cultures - sociales, religieuses, ethniques -, la République, l'humanité. Autant d'appartenances que la notion de fraternité, dans ces différents sens, intègre et questionne. Parmi différents dispositifs d'éducation à la fraternité, je m'intéresse à la Discussion à Visée Philosophique (DVP) en communauté de recherche telle qu'elle a été pensée par ses fondateurs (Lipman; Tozzi) et telle qu'elle est promue par la récente création d'une Chaire de l'UNESCO ${ }^{23}$ dédiée à cette pratique pour les enfants de 5 à 15 ans. L'enjeu de ma recherche est de montrer que la DVP $^{24}$ est, dans le champ de l'éducation à la citoyenneté, un dispositif efficient ${ }^{25}$ pour «faire partager » les valeurs de la République, notamment la fraternité, parce qu'elle permet de les interroger, de les éprouver et de les vivre en acte.

La DVP s'inscrit au cœur du projet d'une authentique éducation à la citoyenneté en prenant le risque d'interroger la valeur des valeurs avec les élèves et par là même les enjeux d'un contrat social dont les futurs citoyens héritent sans l'avoir signé (dimension intellectuelle). N'est-ce pas le seul moyen de rendre possible, dans une démarche co-réflexive, une libre adhésion sans chercher à assujettir les élèves à des injonctions normatives par ailleurs contre-productives? N'est-ce pas la seule voie qui permette de s'emparer de la " propimposition ${ }^{26}$ » de l'EMC tout en évitant les risques d'instrumentalisation idéologique (Tozzi, 2018) ? L'approche philosophique, en plus des habiletés de pensée qu'elle mobilise ${ }^{27}$, et sans attendre la classe Terminale du lycée, ouvre un indispensable champ de conceptualisation et de problématisation, 
notamment dans le domaine moral et politique que l'approche historique, souvent dominante en EMC, ne suffit pas à éclairer. Elle développe ainsi l'esprit critique et le discernement pour s'inscrire parfaitement dans la «culture du jugement» et du « discernement » qui permet la formation laïque de la personne et du citoyen.

Si cette pratique permet de nourrir la dimension intellectuelle d'un travail éducatif sur les valeurs, elle représente également une précieuse ressource pour cultiver les deux autres dimensions - psycho affective et conative -, sans doute les plus importantes si l'on considère que l'EMC vise l'acquisition de dispositions pratiques et effectives. Ma recherche s'attache donc à montrer que la DVP permet, non seulement de " comprendre ", mais aussi et surtout "d'éprouver » et même de "vivre en acte » ces valeurs. La DVP s'inscrit parfaitement dans les finalités du programme d'EMC parce qu'elle incarne en réalité les mêmes valeurs que celles de la République - liberté, égalité, fraternité, laïcité -. Je me limite ici toutefois à l'analyse menée dans ma recherche sur la notion de fraternité pour montrer que la structure de la DVP est porteuse de contraintes spécifiques qui génèrent des dispositions éthiques « fraternelles ». Car l'ambition de " développer des relations de fraternité ${ }^{28}$ » ne saurait se satisfaire de la seule dimension intellectuelle d'une réflexion critique sur les valeurs : il y faut ajouter la dimension psycho-affective et conative si l'on veut espérer les rendre désirables (Kahn, 2016) et susciter une adhésion. Or «le travail philosophique en situation de discussion collective peut être le terreau de sentiments éthiques " (Hawken, 2016, p. 501): le respect de la personne, le tact, la tolérance, l'empathie, la conscience de l'utilité d'une action solidaire, le sentiment d'appartenance. Autant de «marqueurs de fraternité » repérables au sein du dispositif des « ateliers philo » euxmêmes et qui témoignent de ces dispositions éthiques en construction. Mon travail de recherche s'oriente donc dans deux directions: il s'attache, d'une part et principalement, à identifier des marqueurs de fraternité dans les dispositifs de DVP (dimension psycho-affective et conative); d'autre part, à élaborer des outils qui permettent de mener des DVP sur le thème de la fraternité (dimension intellectuelle).

\section{Les conditions méthodologiques de la recherche}

37 La recherche doctorale en cours vise à identifier les indicateurs repérables de ces dispositions éthiques en construction dans le cadre du dispositif - son agencement spatial et temporel, ses règles - comme dans les propos et les attitudes des élèves discutants ou des adultes formés à l'animation de ce type de discussion. Ce travail de recherche s'inscrit dans le sillage d'une sociologie compréhensive et qualitative qui privilégie la démarche inductive (Kaufmann, 2011) ; l'objectif est d'élaborer une grille de « marqueurs de fraternité » à partir de notre matériel d'expérimentation, comme un outil possible dont les futures recherches pourraient s'emparer pour tenter d'évaluer la portée éthique de la DVP. Les marqueurs de fraternité qui ont été l'objet des recueils de données sur le terrain sont les suivants: l'écoute, la connaissance et l'estime de soi et d'autrui, le tact, l'empathie (cognitive et émotionnelle), la tolérance et l'ouverture d'esprit, la solidarité, le sentiment d'appartenance (à la communauté des philosophes, à l'humanité). J'ai privilégié une approche par triangulation (Mucchielli, 2009) en combinant plusieurs sources - observations, questionnaires, entretiens - afin d'obtenir des informations provenant de lieux et de sujets différents. 

REP+ qui ont suivi toute l'année des DVP animées par Johanna Hawken, une praticienne et chercheuse très expérimentée. J'ai observé les discussions dans une classe de $\mathrm{CP}$ composée de 12 élèves qui découvraient la pratique et dans une classe de CM2 composée de 24 élèves qui avaient déjà pratiqué la DVP durant leur parcours scolaire. J'ai observé, filmé et enregistré les séances hebdomadaires de cette classe, puis procédé à la transcription des verbatims de 20 séances. J'ai mené un entretien semi-directif avec l'enseignante ainsi qu'avec Johanna Hawken. Des entretiens-bilan par groupe de 5 élèves ont été réalisés en fin d'année dans la classe de CM2.

Le second terrain était constitué par des classes (de la grande section de maternelle à la $3^{\text {ème }}$ en territoire REP+) qui participent au projet Philojeunes développé dans l'académie de Versailles depuis 2018 par le Centre Académique aux Écoles et aux Établissements (CAAEE) auquel j'appartiens. En tant que chercheur associé au projet, j'ai suivi la formation des 25 enseignants du premier et du second degré à la pratique de la DVP par des praticiens, chercheurs et formateurs historiques - Michel Tozzi, Jean-Charles Pettier, Edwige Chirouter -. J'ai pu observer tout au long de l'année 32 ateliers des 215 pratiqués dans leur classe par les enseignants formés. Un questionnaire de 15 questions ouvertes et fermées a été élaboré pour interroger 98 élèves des collèges qui ont expérimenté la DVP à Gennevilliers (92) et à Grigny (91). Deux entretiens collectifs de bilan ont été réalisés en avril auprès de deux classes de CE1 à Étampes (91) et de CE2 à Grigny (91) pour interroger les élèves sur cette expérience. Durant la même période des entretiens semi-directifs ont été réalisés avec 11 enseignants qui ont participé aux projets. L'évaluation des 18 dossiers pédagogiques mis à disposition par PhiloJeunes pour aider les enseignants à animer leurs séances a fait l'objet d'un questionnaire de 16 questions ouvertes et fermées auprès des enseignants. Enfin j'ai réalisé, avec Stéphanie Miraut, pilote du projet sur l'académie de Versailles pour le CAAEE, l'un des 18 dossiers pédagogiques consacré à la fraternité.

\section{Les marqueurs de fraternité repérables dans la DVP (la forme)}

Les premiers résultats montrent que la Discussion à Visée Philosophique, à condition d'être pratiquée régulièrement - de préférence de façon hebdomadaire et toute l'année - et par des enseignants suffisamment formés à cette pratique, donne l'occasion aux élèves de vivre des expériences qui développent des dispositions éthiques et citoyennes que l'on peut subsumer sous le concept de fraternité tel que notre première partie théorique l'a travaillé et défini : l'écoute, l'estime et la connaissance de soi et des autres, le tact, l'empathie cognitive et émotionnelle, la tolérance et l'ouverture d'esprit, la solidarité, le sentiment d'appartenance. Comment l'expliquer? Ces effets sont produits par la conjonction de deux facteurs: l'objet de la réflexion philosophique - et les contraintes intellectuelles qu'elle génère -, mais aussi les conditions éthiques d'une véritable discussion philosophique en communauté de recherche.

La philosophie propose de réfléchir à des questions existentielles et universelles - la liberté, le bonheur, la mort, le temps qui passe, etc. - qui autorisent la juxtaposition de plusieurs réponses également crédibles. Il n'existe en effet pas une seule réponse unique et définitive à la question : le bonheur dépend-il de nous? Le fait de considérer les enfants comme des interlocuteurs et des penseurs valables, en l'absence de toute évaluation - chose assez rare à l'école - et sans attendre d'eux «la» bonne réponse, 
modifie le statut de l'erreur et produit des effets observables en termes d'estime de soi, notamment chez les élèves habituellement en difficulté scolaire. Ils redoutent moins de se tromper et s'autorisent à penser en première personne. C'est même un des effets les plus manifestes de cette pratique au dire des enseignants dont certains découvrent littéralement leurs élèves sous un jour qu'ils ne soupçonnaient pas. La DVP modifie la nature de la relation qui s'instaure alors entre les élèves, entre les élèves et l'enseignant, offrant au passage un paradigme éthique et épistémique d'autorité éducative dont l'horizontalité bénéficie à tous : il invite les élèves à une co-construction des savoirs tout en conduisant l'enseignant à une pédagogie de la question et du retrait. L'universalité du questionnement, l'impossible clôture des problèmes posés, la pluralité des réponses possibles donnent enfin l'occasion d'une expérience de la commune vulnérabilité de tout être humain face à la grandeur de questions qui ne trouvent pas de réponse unique et définitive. Elle engendre des conduites de solidarité lorsque les élèves se rendent compte qu'ils ne vont pas pouvoir prendre en charge le poids d'une telle question individuellement et qu'elle requiert une coopération de tous les membres du groupe. Cette vulnérabilité, ce pluralisme, l'implication existentielle d'une réponse portée à la première personne conduisent progressivement les élèves à développer des qualités d'empathie à la fois émotionnelle et cognitive. L'analyse des verbatim montre comment cette dernière se manifeste dans l'intrication dialogique des pensées qui émerge après quelques ateliers philo; ce qui n'est souvent d'abord qu'une juxtaposition d'opinions émises à la première personne devient alors un véritable dialogue avec les autres élèves.

Toutefois le seul objet des thématiques philosophiques ne suffit pas à garantir le développement de ces dispositions. Elles ne peuvent advenir qu'à certaines conditions inhérentes au cadre éthique de la discussion : l'écoute, le tact, le respect de la personne distinguée de la valeur de ses propos. C'est là le rôle des règles de la discussion l'interdit fondateur de la moquerie, les règles de prises et de tour de parole, le droit de parler comme de se taire - tout comme des rituels qui sacralisent le temps et l'espace de la discussion - le cercle qui organise la discussion dans un face à face des visages, l'usage d'un bâton de parole, un rituel de début et de fin de discussion, voire une pratique de l'attention -. Car une pensée ne peut être authentiquement philosophique si elle n'est pas à la fois critique et bienveillante, (Lipman, 2003). «La bienveillance est une attitude émotionnelle essentielle pour la pratique philosophique. Pour philosopher, il faut prendre soin les uns des autres" (Hawken, 2019, p. 150). Ainsi, le cadre éthique de la discussion est-il inséparable du contenu philosophique de la discussion: on ne dialogue en commun sur des questions universelles et existentielles qui autorisent une pluralité de positions philosophiques qu'à la condition d'être écouté.e, respecté.e, compris.e, quand bien même on ne partage pas les raisons d'autrui. C'est bien même cette expérience de l'identité commune d'une humanité partagée, y compris dans la différence des désaccords, qui conduit à faire de la Discussion à Visée Philosophique une expérience en acte de la tolérance. Identité: parce qu'elle rapproche les corps et les esprits dans le partage des idées et des émotions. Différence: parce qu'elle est l'occasion d'une pratique qui habitue les élèves, dès le plus jeune âge, à supporter (tolerare) leurs différences sans les transformer de facto en exclusions ; elle permet plutôt de les apprivoiser pour les discuter dans un indispensable travail à la fois rationnel et sensible. Favoriser la connaissance de soi et de l'autre, apprendre à confronter pacifiquement les opinions dans le cadre régulé d'une éthique de la discussion, c'est déjà développer des dispositions à une fraternité laïque. C'est 
permettre aussi le passage progressif de la connaissance de soi à l'altérité, puis de l'altérité à l'universalité en articulant les diverses appartenances qui traversent les individus en construction.

Ainsi l'activité philosophique initie les enfants à l'universalité d'une rationalité questionnante qui leur permet de "se découvrir fils et filles des mêmes questions fondamentales » (Galichet, 2000, p. 18) sans pour autant leur imposer le dogmatisme d'une croyance ou d'une pensée unique. Elle rend tout au contraire possible un pluralisme de positions qui ouvre l'esprit au-delà de la seule position traditionnelle du maitre, travaille la décentration et renforce ainsi l'accès à un point de vue généralisé. Ce faisant, la communauté de recherche nourrit un sentiment d'appartenance à la communauté des citoyens du monde délibérants au cœur d'une rationalité universelle ${ }^{29}$ et au-delà des communautés familières d'appartenance.

\section{Discuter philosophiquement les enjeux de la fraternité (le fond)}

Si la DVP peut, dans sa forme, développer des dispositions éthiques «fraternelles » en tant que dispositif de dialogue philosophique, elle le peut également en interrogeant, dans son contenu, la notion de fraternité et ses enjeux. Un des chantiers de ma recherche m'a donc conduit à élaborer, à l'aide de Stéphanie Miraut, pilote du projet PhiloJeunes dans l'Académie de Versailles, un dossier pédagogique ${ }^{30}$ et des modules de formation ${ }^{31}$ sur le thème de la fraternité. Destinés à des facilitateurs d'« ateliers philo " et constitués de supports divers - albums de littérature de jeunesse, films, séries, affiches, jeux -, ces supports pédagogiques ${ }^{32}$ visent à explorer le champ de questionnement des deux principaux domaines de la fraternité - humaniste et communautaire -. Ils doivent conduire les élèves à interroger la dimension collective de leurs différentes appartenances: la classe, l'établissement, la famille, le genre, les cultures - sociales, religieuses, ethniques - la République, l'humanité.

La réflexion sur les enjeux philosophiques de la fraternité permet par ailleurs de prendre en charge des thématiques sensibles en milieu scolaire : la laïcité, les théories du complot, la prévention des phénomènes de harcèlement entre pairs, les relations fille/garçon. Autant de questions vives qui font l'objet de commandes institutionnelles pour lesquelles les enseignants sont souvent démunis. L'expérience montre qu'une entrée par la fraternité permet d'interroger les fondements problématiques de ces thématiques, mais sans les aborder frontalement. Ainsi, une réflexion philosophique sur les mécanismes d'inclusion-exclusion inhérent à toute logique d'appartenance communautaire permet d'interroger avec profit les soubassements du phénomène de bouc-émissaire à l'œuvre dans les situations de harcèlement. De même, questionner les conditions d'une cohabitation entre nos différentes familles d'appartenances ethniques, sociales, religieuses - permet une entrée moins épidermique dans le champ problématique de la laïcité. Cette approche peut s'avérer bénéfique pour traiter de notions rebattues auprès des élèves et qui génèrent parfois chez eux un mélange de méfiance et de lassitude.

\section{Conclusion}

La fraternité n'est pas seulement l'oubliée du triptyque républicain, elle est aussi l'impensée de l'École. Elle l'a du moins été jusqu'à ce que le contexte sociétal de 
«l'après Charlie» et l'apparition d'un nouveau programme d'EMC ne signalent le besoin d'un nouveau paradigme éducatif que je traduis en une idée-force : il est urgent de penser une culture de la fraternité. L'objectif de ma recherche est de montrer que la pratique de la philosophie à l'école est un dispositif particulièrement efficient pour prendre en charge ce besoin éducatif, social et politique.

On pourrait s'étonner de constater qu'une République qui a fait le choix historique de la laïcité doive paradoxalement s'appuyer sur des «techniques de croyance " pour générer une adhésion à ses valeurs? Le médiologue n'est pas surpris, lui qui ne confond pas le doigt et la lune, distingue le meta et l'inter, c'est-à-dire le principe supérieur d'attachement et les moyens du culte. Une chose est de promouvoir un horizon laïque ${ }^{33}$ dont le meta « idéomoral » est inclus dans le champ de la rationalité issu des Lumières, une autre est de générer une adhésion à ce projet.

N’est-il pas dès lors légitime qu'une éducation citoyenne puisse opposer de puissantes expériences collectives, affectives et rationnelles aux fraternités des pessimismes, entendons par là les embrigadements particulièrement efficaces des théories du complot, des replis identitaires, obscurantistes et populistes? Si, comme le montre Lordon, il semble vain de combattre des opinions par des idées désaffectées ${ }^{34}$, il semble au contraire nécessaire de contribuer au retour en puissance d'un certain nombre de bons sentiments dans le champ agonistique des axiologies pour lutter contre une anthropologie hobbesienne des passions tristes qui naturalise la violence et produit chaque jour des effets sociaux, économiques, politiques et écologiques contestables.

Toutefois, pas plus que l'idée de chien ne mort, le seul appel incantatoire à l'idée de fraternité ne fait communier si elle ne s'incarne pas dans des pratiques. De la même façon, l'appel à la rationalité délibérante du futur citoyen ne suffit pas à former une pensée raisonnable (Lipman, 2003). La société doit pour cela développer « en son sein des enclaves particulières, des univers spéciaux dont les participants se renforcent, par entr'affection, dans l'adhésion à la valeur de rationalité et la cultivent de concert autant qu'ils peuvent " (Lordon, 2016, p. 33). La DVP remplit à cet égard une double fonction : elle cultive la fraternité en acte tout en éduquant à une citoyenneté laïque par le développement d'un affect commun de la rationalité. Elle prend ainsi sa part dans cette volonté de développer des « biotopes concrets de la vertu » (Lordon, 2015, p. 306), ici des communautés empathiques qui, grâce au plaisir de penser en commun, contribuent à une éducation à la fraternité des raisons. Cette pratique s'inscrit pleinement dans le projet laïque de la République démocratique française, laquelle, dans son appel citoyen à l'humanisme issu des Lumières, a établi la rationalité et la fraternité comme des valeurs, mais peine à les incarner.

\section{BIBLIOGRAPHY}

Bergson, H. (2012 [1932]). Les deux sources de la morale et de la religion, chapitre III, « la religion dynamique ». Paris : Flammarion, 2012. 
Bidar, A. (2015). Plaidoyer pour la fraternité. Paris : Albin Michel.

Budex, C. (1999). La fraternité : bref traité du désenchantement et de la férocité, Mémoire de Master sous la direction d'Éric Blondel. Paris I Sorbonne.

Budex, C. (2019). Pratique de la philosophie et fraternité : un levier pour lutter contre les inégalités, Éducation et socialisation, 53, 2019. http://journals.openedition.org/edso/7242 ; DOI: $10.4000 /$ edso.7242

Borgetto, M. (1993). La notion de fraternité en droit public français : le passé, le présent et l'avenir de la solidarité. Paris : LGDJ.

Catheline, N. (2015). Le harcèlement scolaire. Paris : PUF.

Céline (1952 [1932]). Voyage au bout de la nuit. Paris :

Damasio, A. (1994). L'erreur de Descartes et Spinoza avait raison. Paris : Odile Jacob.

Debray, R. (2005). Les communions humaines. Paris : Fayard.

Debray, R. (2009). Le moment fraternité. Paris : Gallimard.

Dumez, H. (2016). Méthodologie de la recherche qualitative : les dix questions clés de la démarche compréhensive. Paris : Vuibert.

Durkheim, E. (1912). Les formes élémentaires de la vie religieuse. Paris : P.U.F.

Galichet, F. (2000). Philosophie et éducation à la citoyenneté. Edição Apf - Associação de Professores de Filosofia.

Garcia, T. (2016). Nous. Paris : Livre de poche 2016.

Gueguen, C. (2018). Heureux d'apprendre à l'école. Paris : Les Arènes.

Hawken, J. (2016). Philosopher avec les enfants : enquête théorique et expérimentale sur une pratique de l'ouverture d'esprit. Thèse de Doctorat. Université Paris-I.

Hawken, J. (2019). 1,2,3 pensez! Philosophons les enfants! 10 règles d'or et outils pédagogiques. Lyon : Chronique sociale.

Kahn, P. (2016). L'esprit du nouvel enseignement moral et civique. In M. Fabre, B. Frelat-Kahn et A. Pachod (dir.). (2016). L'idée de valeur en éducation. Paris : Hermann.

Kaufmann, J.F. (1996). L'entretien compréhensif. Paris : Nathan.

Lipman, M. (2003). À l'école de la pensée. Bruxelles : De Boeck.

Lordon, F. (2015). Imperium. Structures et affects des corps politiques. Paris : La Fabrique.

Lordon, F. (2016). Les affects de la politique. Paris : Seuil.

Levi-Strauss, C. (1983). Le regard éloigné. Paris : Plon.

Marsollier, C., (2016). L'éthique relationnelle, une boussole pour l'enseignant. Paris : Canope.

Marsollier, C. (2017). Les conditions du bien-être à l'école. Économie et Management, Le bonheur au travail, $n^{\circ} 162$, janvier 2017. https://cdn.reseau-canope.fr/archivage/valid/N-9471-14059.pdf

Ménissier, T. (2012). République et fraternité. Une approche de théorie politique. In C. Brice ; G. Bertrand; G. Montègre (dir.). Fraternité. Pour une histoire du concept, Cahiers du " Centre de recherche en histoire et histoire de l'art. Italie, pays alpins ", 20, p. 35-51.

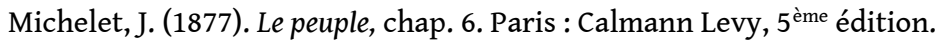


Mucchielli, A. (2009). Dictionnaire des méthodes qualitatives en sciences humaines et sociales. Paris : Armand Colin.

Nussbaum, M. (2011). Les émotions démocratiques. Comment former le citoyen au XXIème siècle. Paris : Flammarion.

Prairat, E. (2017). Éduquer avec tact. Paris : ESF.

Ricœur, P. (1983). Temps et récit. L'intrigue et le récit historique. Tome 1. Paris : Seuil.

Rousseau, JJ. (1966 [1762]). Du contrat social. Paris : Garnier Flammarion.

Tisseron, S. (2010). L'empathie au cour du jeu social. Paris : Albin Michel.

Tozzi, M. (2017). Prévenir la violence par la DVP. Bruxelles : Yakapa.be.

Tozzi, M. (2018). Développer le jugement moral et la citoyenneté des élèves par la DVDP. In B. Berton et C. Leleux (dir.). Pratiques de philosophie et enseignement morale et civique à l'école primaire: quelles articulations? Spirale, $\mathrm{n}^{\circ} 62$.

Zanna, O. (2015). Le corps dans la relation aux autres: pour une éducation à l'empathie. Rennes : Presses Universitaires de Rennes.

\section{NOTES}

1. Le format de l'article ne permet pas de développer tous les éléments méthodologiques qui seront présentés dans une thèse en cours au moment de l'écriture. Les données du terrain mobilisées dans cet article seront donc partielles et incomplètes.

2. The length of the article does not allow to develop all the methodological elements which will be fully presented in a thesis due in December 2020. The field data used and presented in this following article will therefore be partial and incomplete.

3. Par exemple Jules Barni, Manuel républicain, (1872), p. 2 : «sans doute la fraternité, qui n'est plus une chose de droit strict, mais de bienveillance et d'amour, dépend plutôt des mœurs que de la législation ».

4. «Gardez-vous bien de n'entendre par fraternité, comme on le fait trop souvent, qu'une sorte de sentiment vague et tout platonique : vous devez y reconnaitre, au contraire, une loi (...) qui régit la constitution même (...) des États républicains. Pour ceux-ci la fraternité n'est pas seulement un luxe, elle est le nécessaire (...) La fraternité est (...) l'essentielle condition de vie pour une société démocratique ", Alfred Fouillée, Discours prononce à l'Assemblée nationale pour la distribution des récompenses (18 juillet 1886), cité in G. Gauthier et C. Nicolet, La laïcité en mémoire, pp. 222-223, 1987, Edilig.

5. Parce qu'elle peut tout aussi bien être ouverte que fermée, la fraternité peut aussi bien être un remède qu'un poison social.

6. Pinotti, A. (2016). L'empathie, Histoire d'une idée de Platon au posthumain. Paris : Vrin.

7. Nous ne rentrons pas ici dans l'analyse des attitudes philosophiques qui légitiment - ou pas la distinction entre ces deux termes (Prairat, 2015). Sans les confondre comme des synonymes, je les considère également utiles pour caractériser la nature des relations humaines, comprise dans leur dimension individuelle, par opposition à la dimension collective des fraternités communautaires. L'expression d'«éthique relationnelle» (Marsollier, 2016) désigne ainsi le domaine de réflexion qui étudie la «manière d'être » (ethos) à l'œuvre dans la relation entre deux personnes lors d'expériences comme la pitié, le visage, la dignité, le tact, l'hospitalité -, autant d'expériences dont les enjeux moraux ne sont pas exempts. 
8. La fraternité est en effet un "principe » juridique dont le conseil constitutionnel a d'ailleurs récemment précisé le sens, le 6 juillet 2018, à propos de l'article L 622-1 du code de l'entrée, du séjour des étrangers et du droit d'asile - rebaptisé Délit de solidarité par ses détracteurs - ; mais elle aussi une "valeur" de la République au sens où elle joue le rôle d'un "idéal commun ", promu comme tel par le préambule de la constitution, et dont l'horizon axiologique ne se limite pas à sa portée juridique.

9. La «fraternité humaniste» peut, elle aussi, entrer dans la catégorie des fraternités communautaires. Elle possède donc elle-même une double dimension selon qu'on la considère dans sa dimension interindividuelle - par exemple au travers des expériences de la pitié chez Rousseau - ou bien dans sa dimension collective. Dans ce dernier cas, elle concerne la question identitaire de l'appartenance à un genre qui s'est auto-proclamé « empire dans un empire ». Cela soulève le problème des frontières (Garcia, 2016) d'une communauté qui, pour se constituer, exclut une partie des êtres vivants, critique que les anti-spécistes ne manquent pas d'adresser à une certaine conception humaniste.

10. Ces trois auteurs, à la fois philosophes et d'ailleurs pères fondateurs de la sociologie, ont en effet en commun d'avoir repéré la dimension religieuse du lien social et politique, c'est-à-dire la nécessité, pour tout groupe, de cultiver une sacralité - qui n'est pas nécessairement divine - pour entretenir et cultiver son sentiment d'appartenance.

11. "Plus un Nous s'étend, moins il est intense ; mais plus il s'intensifie, moins il est étendu » (Garcia, $2016: 212$ ).

12. Manuscrit de Genève, O.C., p. 287, T.III.

13. Discours sur l'économie politique, p. 254, O.C., T.III.

14. Récupéré le 4 décembre 2019 de https://eduscol.education.fr/cdi/actualites/archives/1ersemestre-2015/mesures-valeurs-rep

15. Le programme initial de 2015 (BO spécial $n^{\circ} 6$ du 25 juin 2015) a fait l'objet de plusieurs ajustements pour l'école élémentaire et le collège en 2018 (BO $\mathrm{n}^{\circ} 30 \mathrm{du} 26$ juillet 2018).

16. Récupéré le 4 décembre 2019 de https://cache.media.eduscol.education.fr/file/04_Avril/ 64/5/Rapport_pour_un_enseignement_laique_de_la_morale_249645.pdf

17. Qui conduit à l'action (conatus : élan, tendance).

18. Les modifications de ce programme initial opérées en 2018 pour les cycles 2,3 et 4 n'ont toutefois pas remis en cause la nécessité d'une culture de la sensibilité et de l'engagement.

19. Très souvent confondues, elles se distinguent pourtant: la solidarité renvoie à l'idée d'entraide, de coopération, de défense d'intérêts communs; la fraternité possède une dimension affective et désigne plus spécifiquement un "sentiment d'appartenance ». D'un point de vue philosophique et juridique (Borgetto, 1993), la solidarité doit être comprise comme un sousconcept de l'idée de fraternité.

20. Le BOEN spécial $n^{\circ} 6$ du 25 juin 2015 a été modifié en 2019 pour les classes de seconde et de première (BO spécial $\mathrm{n}^{\circ} 1$ du 22 janvier 2019) ainsi que les classes Terminales des voies générales et technologiques (BO spécial $n^{\circ} 8$ du 25 juillet 2019).

21. De même, un ouvrage intitulé « Les valeurs de la République », écrit par Françoise Martinetti, édité en 2015 par un éditeur institutionnel de l'éducation nationale (Canopé) l'ignore notoirement. Sans faire aucun procès d'intention, on est en droit d'interroger les raisons d'une telle absence.

22. Ainsi les enquêtes locales de climat scolaire montrent que les principales violences vécues par les élèves «trouvent leur ancrage dans le rapport à l'altérité, tout particulièrement dans la nonacceptation de la différence et plus largement, dans la construction identitaire et les valeurs qui la fondent » (Marsollier, 2017 : 39-40).

23. Récupéré le 4 décembre 2019 de https://chaireunescophiloenfants.univ-nantes.fr/

24. Il est notable que l'expression de "Discussion à Visée Philosophique » a été retirée du programme initial de 2015 au profit des expressions de "discussion réglée » ou de "débat 
argumentée » (BO $\mathrm{n}^{\circ} 30$ du 26 juillet 2018). Faut-il voir dans cette rétractation une réticence institutionnelle à voir le terme de "philosophie» associé à une pratique scolaire émancipée d'une tradition universitaire qui cantonnait jusqu'alors cette discipline à la dernière année du lycée ? Elle bénéfice pourtant, depuis les années 70 aux États-Unis (Lipman), ou plus récemment en France (Tozzi), d'un ensemble de recherches qui témoignent du sérieux de sa démarche. Laissant cette querelle de côté, je considèrerai la DVP comme un genre de "discussion réglée ».

25. Voilà pourquoi le programme précise que «la discussion réglée et le débat argumenté ont une place de premier choix pour permettre de comprendre, d'éprouver et de mettre en perspective les valeurs qui régissent notre société démocratique ».

26. On peut repérer une tension ou même une injonction paradoxale dans les attentes d'un programme d'EMC qui semble proposer et imposer à la fois. Il rappelle que «les valeurs et principes de la République fondent le pacte républicain » et que «les transmettre et les faire partager est une œuvre d'intégration républicaine»; de même, il "insiste à la fois sur l'autonomie du citoyen et sur son appartenance à la communauté politique formée autour des valeurs et des principes de la République ", (BO n 30 du 26 juillet 2018).

27. Le nombre et le nom des habiletés de pensée mobilisées par la DVP fait l'objet d'un débat fécond entre chercheurs et praticiens. Michel Tozzi (2018) en retient trois : conceptualiser, problématiser, argumenter.

28. Préambule du programme pour les cycles 2,3 et 4 (BO $n^{\circ} 30$ du 26 juillet 2018).

29. L'enquête menée auprès des 98 collégiens montrent que les enfants sont tout à fait conscients de se poser des questions que tous les enfants du monde se sont toujours posées.

30. Récupéré le 3 novembre 2019 de https://philojeunes.org/philojeunes/le-materielpedagogique/fiches-dvdp/

31. Ces modules de formations ont été dispensés à Toulouse, Lille, Rennes, Paris en 2018 et 2019 dans le cadre de l'association SEVE qui initie à la pratique de la philosophique avec les enfants. Récupéré le 3 décembre 2019 de https://asso.seve.org/wp-content/uploads/sites/2/2018/12/ APPRO_-FRATERNITE-LAICITE_PARIS_2019.pdf

32. Le format de cet article ne permet pas de présenter l'ensemble de ces supports. Je renvoie toutefois à un padlet qui donne un aperçu de certains outils utilisés pour réaliser des cueillettes de questions philosophiques préalables à des DVP. Récupéré le 3 décembre 2019 de https:// padlet.com/cbudex/dvpfraternitesupports

33. Soulignons au passage la tension, voire la contradiction liée à cette "propimposition ». Jaurès avait raison d'affirmer qu' " il n'y a que le néant qui soit neutre ", à moins de se "condamner par là à n'avoir ni doctrine, ni pensée, ni efficacité intellectuelle et morale » (Revue de l'enseignement primaire et primaire supérieur, octobre 1908). Il faut bien comprendre que le transfert de sacralité d'un meta religieux vers un meta laïque est un choix culturel ou civilisationnel.

34. «Il s'agit tout de même que les abstractions idéelles gagnent en pouvoir d'affecter, et qu'elles deviennent capables de faire effet au-delà de cette minorité qu'on appelle usuellement les intellectuelles » (Lordon, 2016, p. 169).

\section{ABSTRACTS}

This article is based on an ongoing $\mathrm{PhD}$ research ${ }^{2}$. It begins by reaffirming the stakes of an education to fraternity by highlighting the issues and difficulties that exist in embodying in concrete actions and practices a value that is yet essential to the political and social life. It then 
goes on to show that philosophically oriented discussion contributes to an education to citizenship because it allows to question, experience and live in action the values of the Republic among which fraternity stands. Recommended from elementary school onwards by the civic and moral teaching program since 2015, this system allows, through the reasoned and regulated discussion of universal and existential questions, the emergence of ethical abilities that contribute to the necessary culture of a secular fraternity.

Cet article s'appuie sur une partie des données d'une recherche doctorale encore en cours ${ }^{1}$. Il rappelle d'abord les enjeux d'une éducation à la fraternité en soulignant les difficultés à incarner dans des pratiques quotidiennes une valeur pourtant indispensable à la vie sociale et politique. Il montre ensuite que la discussion à visée philosophique (DVP) contribue à une éducation à la citoyenneté parce qu'elle permet d'interroger, d'éprouver et de faire vivre en acte les valeurs de la République, notamment la fraternité. Préconisée dès l'école élémentaire par le programme d'Enseignement Moral et civique depuis 2015 ce dispositif permet, par la discussion argumentée et régulée de questions universelles et existentielles, l'émergence de dispositions éthiques qui participent à la nécessaire culture d'une fraternité laïque.

\section{INDEX}

Keywords: education to fraternity, philosophical discussion, moral and civic education, French "laïcité", philosophy for children

Mots-clés: éducation à la fraternité, discussion à visée philosophique, enseignement moral et civique, laïcité, philosophie avec les enfants

\section{AUTHOR}

\section{CHRISTIAN BUDEX}

Université de Nantes, Centre de Recherche en Education de Nantes (CREN) 